\title{
AVALIAÇÃO DO DESEMPENHO E SATISFAÇÃO DOS USUÁRIOS DE PRÓTESES TOTAIS MUCOSSUPORTADAS FORNECIDAS PELO SISTEMA ÚNICO DE SAÚDE EM MUNICÍPIO CATARINENSE
}

\author{
Débora Passos CRISTIANO ${ }^{1}$ \\ E-mail:debycristiano@hotmail.com \\ Andreia COLLODEL ${ }^{2}$ \\ E-mail: collodeland.odonto@hotmail.com
}

Luciane Bisognin CERETTA ${ }^{3}$

E-mail: luk@unesc.net

Priscyla Waleska SIMÕES ${ }^{4}$

E-mail:pri@unesc.net

Renan Antonio CERETTA ${ }^{5}$

E-mail:rce@unesc.net

Fernanda Guglielmi Faustini SONEGO ${ }^{6}$

E-mail:fgfsonego@unesc.net

\section{RESUMO}

O edentulismo ainda é considerado um grande problema para a saúde pública, no Brasil. Acomete parte da população, em regiões distintas e diferentes faixas etárias. Alguns municípios brasileiros aderiram aos Laboratórios Regionais de Próteses Dentárias para minimizar essa condição; assim, buscou-se avaliar o desempenho e a satisfação dos usuários de próteses totais mucossuportadas fornecidas pelo Sistema Único de Saúde. Estudo transversal, de análise descritiva e abordagem quali-quantitativa, com amostra censitária de 115 usuários que receberam próteses totais superiores e/ou inferiores no período de julho de 2013 até julho de 2014. Foram aplicados os questionários OHIP-14 e a Escala Visual Analógica para avaliar

1 Cirurgiã-dentista residente - Programa de Residência Multiprofissional em Atenção Básica/Saúde da Família - Universidade do Extremo Sul Catarinense

2 Cirurgiã-dentista formada pela Universidade do Extremo Sul Catarinense.

3 Doutora em Ciências da Saúde - Universidade do Extremo Sul Catarinense. Professora do Curso de Odontologia e Professora Pesquisadora no Programa de Pós-Graduação em Saúde Coletiva - Universidade do Extremo Sul Catarinense.

4 Doutora em Ciências da Saúde - Universidade do Extremo Sul Catarinense. Professora do Curso de Odontologia e Professora Pesquisadora no Programa de Pós-Graduação em Saúde Coletiva - Universidade do Extremo Sul Catarinense.

5 Doutor em Ciências da Saúde - Universidade do Extremo Sul Catarinense. Professor do Curso de Odontologia -Universidade do Extremo Sul Catarinense.

6 Mestre em Odontologia. Professora do Curso de Odontologia - Universidade do Extremo Sul Catarinense. 
Cristiano DP, Collodel A, Ceretta LB, Simões PW, Ceretta RA, Sonego FGF. Avaliação do desempenho e satisfação dos usuários de próteses totais mucossuportadas fornecidas pelo Sistema Único de Saúde em município catarinense. Rev. Odontol. Univ. Cid. São Paulo 2018 abr/jun 30(2) 116-31

ISSN 1983-5183

a satisfação e desempenho. A idade mediana foi 59,0 (53,0-68,0) anos. Renda mensal prevalente foi de até um salário mínimo $(60,0 \% ; n=69)$. O grau de alfabetização mais comum foi ensino fundamental incompleto (68,7\%; $n=79)$. O desempenho mediano associado aos diferentes tipos de próteses foi de 99,0 para a superior, 95,0 para a inferior e 97,0 para ambas as próteses, resultados considerados estatisticamente significativos $(p=0,047)$. Usuários do SUS que receberam próteses totais mucossuportadas estão satisfeitos com a reabilitação. Quanto ao desempenho das próteses avaliadas, constatou-se diferença estatisticamente significativa para reabilitação com prótese superior, inferior ou para ambas.

DESCRITORES: PRÓTESE TOTAL - SAÚDE BUCAL - ODONTOLOGIA COMUNITÁRIA - SAÚDE PÚBLICA.

\title{
EVALUATION OF PERFORMANCE AND USERS SATISFACTION OF COMPLETE DENTURE PROSTHESIS PROVIDED BY THE UNIFIED HEALTH SYSTEM IN SANTA CATARINA MUNICIPALITY
}

\begin{abstract}
Edentulism is still considered a great problem for public health in Brazil. Affect the population in different regions and different ages. Some cities have joined the Regional Laboratories of Dental Prosthetics to minimize this condition, so it has been intended to evaluate the performance and user satisfaction of complete denture prosthesis provided by the Unified Health System. Cross-sectional study of descriptive analysis and qualitative and quantitative approach, with census sample of 115 users who have received upper and/ or lower complete dentures from July 2013 to July 2014. We applied the OHIP-14 questionnaire and Visual Analog Scale to assess satisfaction and performance. The median age was 59.0 (53.0 to 68.0) years. Prevalent monthly income was up to one minimum wage $(60.0 \% ; n=69)$. The most common grade literacy was incomplete primary education $(68.7 \% ; n=79)$. Average performance associated with different types of prosthesis was 99.0 for higher, 95.0 was lower and 97.0 for both prostheses, results were considered statistically significant $(p=0.047)$. SUS users who have received complete dentures prosthesis are satisfied with the rehabilitation. As for the performance of prosthesis assessed, there was no statistically significant difference for rehabilitation with upper dentures, lower or both.
\end{abstract}

DESCRIPTORS: DENTURE, COMPLETE - ORAL HEALTH - COMMUNITY DENTISTRY - PUBLIC HEALTH.

\section{INTRODUÇÃO}

Problemas bucais afetam diretamente a vida dos indivíduos e podem alterar a normalidade da saúde sistêmica, estando totalmente interligados e considerados transtornos para uma boa condição de vida. Alterações na saúde bucal podem gerar consequências funcionais e psicossociais, modificando o estilo de vida, agindo sobre a saúde sistêmica e comprometendo diretamente as práticas diárias .

Atualmente, o edentulismo ainda é considerado um grande problema para a Saúde Pública, principalmente em determinadas faixas etárias. A perda dentária causa consequências graves para a saúde em geral ${ }^{2,3}$. Muitos profissionais acabam priorizando os tratamentos restauradores e reconstrutores dos elementos dentais e a reabilitação protética torna-se falha, porém fundamental em determinados casos, pois possibilita uma melhora na qualidade de vida².

Mediante resultados da Pesquisa Nacional de Saúde Bucal, referente ao uso e necessidade de prótese odontológica para a população brasileira, o edentulismo é reconhecido como um grande problema, 
ISSN 1983-5183

servindo ao mesmo tempo para subsidiar ações de planejamento a partir da análise das necessidades ${ }^{4}$.

O edentulismo, no Brasil, ainda se encontra presente na população, mas destacado de maneira diferente em regiões distintas e em diferentes faixas etárias. Entre 35 e 44 anos, a utilização de próteses totais é de $14,5 \%$ na Região Sul, percentual mais alto comparado às outras regiões. Já na faixa etária de 65 a 74 anos o percentual chega a 63,1\% de pessoas que utilizam próteses totais em todo o Brasil, proporção considerada alta. Destaca-se novamente a utilização na Região Sul, em que 65,3\% da população utilizam próteses totais ${ }^{4}$. Verifica-se que o edentulismo ainda está muito presente, assim como a necessidade de observação e orientação em especial àqueles que vivem dentro dessas condições ${ }^{4}$.

A Política Nacional de Saúde Bucal propõe várias ações com intuito de facilitar o atendimento odontológico a todos os usuários que acessarem o Sistema Único de Saúde (SUS). As principais ações ${ }^{5,6}$ destacadas são as de promoção e prevenção, a reorganização da Atenção Básica em Saúde Bucal, a qualificação da Atenção Especializada, com a implantação e ampliação dos Centros de Especialidades Odontológicas (CEO) e a reabilitação oral dos usuários com próteses, por meio dos Laboratórios Regionais de Próteses Dentárias (LRPD) implantadas para esse fim. Assim, torna-se possível a reabilitação oral para aqueles que necessitam e não possuem condições financeiras, sendo ela muito importante para melhorar a qualidade de vida dos usuários 5 .

Como muitos municípios brasileiros aderiram aos LRPD, torna-se importante monitorar e, se necessário, adequar o serviço ofertado, bem como verificar a melhoria na qualidade de vida dos reabilitados. Dessa forma, o presente estudo objetiva avaliar o desempenho e a satisfação dos usuários de próteses totais mucossuportadas fornecidas pelo SUS, em um município sul catarinense.

\section{MÉTODOS}

A pesquisa foi realizada em um município sul catarinense que, segundo o Instituto Brasileiro de Geografia e Estatística (IBGE)7, possuía, em 2014, uma população estimada de 52.284 habitantes, após aprovação pelo Comitê de Ética e Pesquisa em Seres Humanos do local onde foi realizada a pesquisa, sob o parecer no 702.911/2014.

Tratou-se de um estudo transversal, de análise descritiva e abordagem quali-quantitativa, envolvendo uma amostra censitária de 250 contemplados; no entanto, devido aos critérios de inclusão e exclusão e às perdas associadas, a amostra final resultou em 115 usuários, que receberam próteses totais mucossuportadas superiores e/ou inferiores, confeccionadas no LRPD do município, no período de julho de 2013 até julho de 2014.

O município possui 13 Estratégias Saúde da Família (ESF) que dispõem de atendimento odontológico básico e que estão localizadas de acordo com bairros de origem. Possui também um CEO, que serve de referência às ESF e realiza algumas especialidades, dentre elas a confecção de próteses totais superiores e/ ou inferiores, mucossuportadas, distribuídas à população de edêntulos.

Foram incluídos na pesquisa indivíduos de ambos os gêneros, que aceitaram e assinaram o Termo de Consentimento Livre e Esclarecido (TCLE), moradores do município, com boa compreensão da língua portuguesa, que receberam a prótese total superior e/ou inferior, e que estavam utilizando-a(s) há pelo menos 3 (três) meses. Foram excluídos os que receberam próteses parciais mucossuportadas, moradores de outros municípios, aqueles que apresentaram desordens do sistema estomatognático, alterações 
ISSN 1983-5183

patológicas dos rebordos alveolares e doenças sistêmicas debilitantes.

Inicialmente, foi feita a análise dos prontuários do CEO para a coleta dos dados dos usuários que receberam próteses totais mucossuportadas confeccionadas no período estipulado pelo estudo e que se encaixavam nos critérios de inclusão e exclusão. Na sequência os usuários foram separados de acordo com a ESF de origem ou com a que encaminhou os pacientes e contatados em suas residências a participarem do estudo.

Assim, foram aplicados dois questionários aos participantes, um estruturado e outro semiestruturado, para avaliarem a satisfação dos usuários na utilização de suas novas próteses totais e o desempenho delas.

Primeiramente foi aplicado o questionário estruturado Ontario Health Insurance Plan (OHIP-14) para avaliar o perfil de impacto da saúde bucal, contendo 14 perguntas relacionadas à limitação funcional, desconforto psicológico, inabilidade física, psicológica e social, dor física e incapacidades ligadas ao uso de próteses totais mucossuportadas. As respostas foram registradas por meio de uma escala de 0 a $4(0=$ nunca; 1 = quase nunca; 2 = ocasionalmente; 3 = algumas vezes; $4=$ sempre) $)^{8}$.

O perfil socioeconômico também foi questionado, possibilitando uma avaliação e correlação de fatos necessários para o entendimento dos resultados do estudo.

O segundo questionário semiestruturado avaliou, por meio da Escala Visual Analógica (EVA), o nível de satisfação dos pacientes, de acordo com o desempenho, das próteses ${ }^{9}$.

Os sujeitos foram instruídos a marcar uma linha perpendicular à reta EVA, correspondente ao seu nível de satisfação de desempenho, que foi medido em milímetros da esquerda da linha até a marcação correspondente pelo participante. A pontuação foi registrada sendo que a escala é quantificada de 0

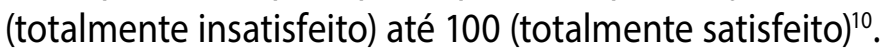

Por meio desse questionário, adaptado de Regis ${ }^{11}$ (2012), objetivou-se avaliar a satisfação dos usuários relacionada à sua condição bucal, facilidade de remoção da prótese, conforto e estética. Também foi avaliada a percepção dos participantes referente ao desempenho das próteses totais durante diversas funções, como fala, mastigação e movimentação da língua, assim como a facilidade na adaptação, habilidade de limpeza, habilidade durante a mastigação de alguns alimentos específicos como cenoura crua, bife, alface, maçã, queijo, pão fresco, sendo diferenciados por possuírem dureza e texturas diferentes. Os participantes puderam avaliar também a qualidade da mastigação desses alimentos na EVA, ancorados com as palavras "mal mastigadas" e "muito bem mastigadas".

Após a finalização da coleta de dados, foi realizada a análise estatística no software Statistical Package for the Social Sciences (SPSS), versão 22.0. Foi testada a normalidade de todas as variáveis numéricas pelo teste de Kolmogorov-Smirnov, que revelou distribuição não gaussiana; assim, foi calculada a mediana e o intervalo interquartil para essas variáveis. Também foi estimada a frequência absoluta (n) e relativa (\%) para as variáveis qualitativas.

Foi utilizado o teste Kruskal Wallis para analisar a relação entre o tipo de prótese e todas as demais variáveis numéricas, sucedido do teste de Dunn em caso de significância estatística e, também, para avaliar a mediana da satisfação geral em relação à escolaridade. $O$ teste de qui-quadrado de Pearson foi utilizado para avaliar a associação entre escolaridade e renda mensal, e entre o tipo de prótese e o questionário OHIP-14. Utilizamos, também, o teste $U$ de Mann Whitney, buscando avaliar a satisfação geral em relação 
ISSN 1983-5183

ao gênero. Foi realizada a correlação de Spearman entre a idade e o grau de satisfação geral. Para todos os testes supracitados foram considerados como significativos $\alpha=0,05$ e intervalo de confiança de $95 \%$.

\section{RESULTADOS}

Participaram da pesquisa 115 pacientes, considerando os critérios de inclusão e exclusão, 68,7\% mulheres $(n=79)$ e $31,3 \%$ homens $(n=36)$. A idade mediana foi de $59,0(53,0-68,0)$ anos. Renda mensal mais frequente foi de até um salário mínimo $(60,0 \% ; n=69)$. Na Tabela 1, são apresentados os dados referentes ao perfil da amostra.

Tabela 1 - Perfil dos participantes que receberam próteses totais mucossuportadas num município sul catarinense, entre julho de 2013 e julho de 2014.

\begin{tabular}{ll}
\hline \hline Variável & $\mathrm{n}(\%)$ \\
& $\mathrm{n}=115$ \\
\hline Idade mediana (Intervalo interquartil) & $59,0(53,0-68$ \\
Gênero & \\
Feminino & $79(68,7)$ \\
Masculino & $36(31,3)$ \\
& \\
Estratégia Saúde da Família (ESF) & \\
ESF PM & $25(21,7)$ \\
ESF PV & $8(7,0)$ \\
ESF L. & $13(11,3)$ \\
ESF CR & $15(13,0)$ \\
ESF J. & $4(3,5)$ \\
ESF 3L & $3(2,6)$ \\
ESF A. & $17(14,8)$ \\
ESF VN1 e VN2 & $3(2,6)$ \\
ESF BV & $6(5,2)$ \\
ESF E & $11(9,6)$ \\
ESF JE & $7(6,1)$ \\
ESF NSF & $3(2,6)$
\end{tabular}

Renda Mensal do paciente

Não possui

$30(26,1)$

Até 1 salário mínimo

$69(60,0)$

De 1 a 2 salários

$16(13,9)$ 
ISSN 1983-5183

Variável

$\mathrm{n}(\%)$

$\mathrm{n}=115$

Escolaridade

Nunca estudou

Ensino fundamental incompleto

Ensino fundamental completo

$12(10,4)$

Ensino médio incompleto

$8(7,0)$

Ensino médio completo

Tempo mensal mediano de utilização das próteses (Intervalo Interquartil)

$12,0(8,0-12,0)$

Próteses utilizadas

Superior

$66(57,4)$

Inferior

Ambas

$47(40,9)$

A Tabela 2 mostra a satisfação dos pacientes quanto à utilização de próteses totais mucossuportadas diante de questões sociais, biológicas e psicológicas.

Sobre a dificuldade de pronunciar as palavras, $75,8 \%(n=50)$ daqueles que utilizaram prótese superior responderam que nunca tiveram algum problema, assim como a maioria dos que utilizaram ambas as próteses, $70,2 \%(n=33)$.

Sobre sentir o gosto dos alimentos, $83,3 \%(n=55)$ responderam que nunca encontraram dificuldade em sentir o gosto com a utilização de prótese superior apenas, e quanto ao fato de terem que interromper as refeições por causa da utilização de prótese superior, $72,7 \%(n=48)$ responderam nunca e 3,0\% ( $n=2)$ sempre tiveram que parar de se alimentar.

Quando questionados sobre a vida ser menos insatisfatória devido aos problemas relacionados com a prótese superior, $90,9 \%(n=60)$ relataram nunca sentir tal insatisfação, enquanto os que utilizam próteses inferiores, $100,0 \%(n=2)$ concordaram, e os que utilizam ambas as próteses $89,4 \%(n=42)$ responderam nunca.

Referente à influência da saúde bucal sobre a saúde sistêmica, 53,0\% ( $n=61)$ afirmam conhecer essa relação e $47,0 \%$ ( $n=54$ ) desconhecem e, quando questionados sobre o motivo dessa influência, 17,4\% ( $n=20)$ relataram problemas com a utilização das próteses antigas que acabaram afetando a sua saúde sistêmica. Dos $19,1 \%(n=22)$ dos pacientes, que referiram problemas com as próteses e que no questionário foram classificados como outros, 9,6\% ( $n=11)$ salientaram que a prótese inferior não deu certo e $5,2 \%(n=6)$ que os dentes descolaram com facilidade. 
ISSN 1983-5183

Tabela 2 - Características encontradas no questionário OHIP-14.

\begin{tabular}{|c|c|c|c|c|}
\hline \multirow[t]{2}{*}{ Variável } & & Prótese Superior & Prótese Inferior & Ambas \\
\hline & & $\mathrm{n}=66$ & $\mathrm{n}=2$ & $n=47$ \\
\hline \multirow[t]{5}{*}{ Dificuldade em pronunciar palavras } & Nunca & $50(75,8)$ & $2(100,0)$ & $33(70,2)$ \\
\hline & Quase nunca & $11(16,7)$ & $0(0,0)$ & $6(12,8)$ \\
\hline & Ocasionalmente & $0(0,0)$ & $0(0,0)$ & $2(4,3)$ \\
\hline & Algumas vezes & $3(4,5)$ & $0(0,0)$ & $5(10,6)$ \\
\hline & Sempre & $2(3,0)$ & $0(0,0)$ & $1(2,1)$ \\
\hline \multirow{5}{*}{$\begin{array}{l}\text { Dificuldade de sentir gosto dos ali- } \\
\text { mentos }\end{array}$} & Nunca & $55(83,3)$ & $2(100,0)$ & $33(70,2)$ \\
\hline & Quase nunca & $7(10,6)$ & $0(0,0)$ & $7(14,9)$ \\
\hline & Ocasionalmente & $2(3,0)$ & $0(0,0)$ & $3(6,4)$ \\
\hline & Algumas vezes & $1(1,5)$ & $0(0,0)$ & $2(4,3)$ \\
\hline & Sempre & $1(1,5)$ & $0(0,0)$ & $2(4,3)$ \\
\hline \multirow[t]{5}{*}{ Dor em sua boca } & Nunca & $26(39,4)$ & $0(0,0)$ & $22(46,8)$ \\
\hline & Quase nunca & $28(42,4)$ & $1(50,0)$ & $14(29,8)$ \\
\hline & Ocasionalmente & $1(1,5)$ & $0(0,0)$ & $4(8,5)$ \\
\hline & Algumas vezes & $9(13,6)$ & $0(0,0)$ & $4(8,5)$ \\
\hline & Sempre & $2(3,0)$ & $1(50,0)$ & $3(6,4)$ \\
\hline \multirow{5}{*}{$\begin{array}{l}\text { Desconforto ao comer algum alimen- } \\
\text { to }\end{array}$} & Nunca & $27(40,9)$ & $0(0,0)$ & $19(40,4)$ \\
\hline & Quase nunca & $25(37,9)$ & $1(50,0)$ & $15(31,9)$ \\
\hline & Ocasionalmente & $2(3,0)$ & $0(0,0)$ & $4(8,5)$ \\
\hline & Algumas vezes & $10(15,2)$ & $0(0,0)$ & $5(10,6)$ \\
\hline & Sempre & $2(3,0)$ & $1(50,0)$ & $4(8,5)$ \\
\hline \multirow[t]{5}{*}{ Sentiu-se preocupado } & Nunca & $50(75,8)$ & $0(0,0)$ & $28(59,6)$ \\
\hline & Quase nunca & $10(15,2)$ & $2(100,0)$ & $13(27,7)$ \\
\hline & Ocasionalmente & $1(1,5)$ & $0(0,0)$ & $0(0,0)$ \\
\hline & Algumas vezes & $4(6,1)$ & $0(0,0)$ & $4(8,5)$ \\
\hline & Sempre & $1(1,5)$ & $0(0,0)$ & $2(4,3)$ \\
\hline \multirow[t]{5}{*}{ Sentiu-se tenso } & Nunca & $52(78,8)$ & $0(0,0)$ & $29(61,7)$ \\
\hline & Quase nunca & $8(12,1)$ & $2(100,0)$ & $12(25,5)$ \\
\hline & Ocasionalmente & $3(4,5)$ & $0(0,0)$ & $0(0,0)$ \\
\hline & Algumas vezes & $3(4,5)$ & $0(0,0)$ & $5(10,6)$ \\
\hline & Sempre & $0(0,0)$ & $0(0,0)$ & $1(2,1)$ \\
\hline
\end{tabular}


ISSN 1983-5183

\begin{tabular}{|c|c|c|c|c|}
\hline \multirow[t]{2}{*}{ Variável } & & Prótese Superior & Prótese Inferior & Ambas \\
\hline & & $\mathrm{n}=66$ & $\mathrm{n}=2$ & $\mathrm{n}=47$ \\
\hline \multirow[t]{5}{*}{ Alimentação foi insatisfatória } & Nunca & $45(68,2)$ & $1(50,0)$ & $23(48,9)$ \\
\hline & Quase nunca & $13(19,7)$ & $1(50,0)$ & $15(31,9)$ \\
\hline & Ocasionalmente & $2(3,0)$ & $0(0,0)$ & $2(4,3)$ \\
\hline & Algumas vezes & $3(4,5)$ & $0(0,0)$ & $7(14,9)$ \\
\hline & Sempre & $3(4,5)$ & $0(0,0)$ & $0(0,0)$ \\
\hline \multirow[t]{5}{*}{ Teve que interromper refeições } & Nunca & $48(72,7)$ & $1(50,0)$ & $25(53,2)$ \\
\hline & Quase nunca & $11(16,7)$ & $1(50,0)$ & $14(29,8)$ \\
\hline & Ocasionalmente & $1(1,5)$ & $0(0,0)$ & $4(8,5)$ \\
\hline & Algumas vezes & $4(6,1)$ & $0(0,0)$ & $2(4,3)$ \\
\hline & Sempre & $2(3,0)$ & $0(0,0)$ & $2(4,3)$ \\
\hline \multirow[t]{5}{*}{ Dificuldade para relaxar } & Nunca & $56(84,8)$ & $1(50,0)$ & $31(66,0)$ \\
\hline & Quase nunca & $5(7,6)$ & $0(0,0)$ & $7(14,9)$ \\
\hline & Ocasionalmente & $1(1,5)$ & $0(0,0)$ & $3(6,4)$ \\
\hline & Algumas vezes & $3(4,5)$ & $1(50,0)$ & $3(6,4)$ \\
\hline & Sempre & $1(1,5)$ & $0(0,0)$ & $3(6,4)$ \\
\hline \multirow[t]{5}{*}{ Sentiu-se envergonhado } & Nunca & $60(90,9)$ & $2(100,0)$ & $35(74,5)$ \\
\hline & Quase nunca & $3(4,5)$ & $0(0,0)$ & $8(17,0)$ \\
\hline & Ocasionalmente & $0(0,0)$ & $0(0,0)$ & $2(4,3)$ \\
\hline & Algumas vezes & $3(4,5)$ & $0(0,0)$ & $1(2,1)$ \\
\hline & Sempre & $0(0,0)$ & $0(0,0)$ & $1(2,1)$ \\
\hline \multirow[t]{5}{*}{ Ficou irritado com outras pessoas } & Nunca & $63(95,5)$ & $2(100,0)$ & $41(87,2)$ \\
\hline & Quase nunca & $2(3,0)$ & $0(0,0)$ & $2(4,3)$ \\
\hline & Ocasionalmente & $0(0,0)$ & $0(0,0)$ & $2(4,3)$ \\
\hline & Algumas vezes & $1(1,5)$ & $0(0,0)$ & $1(2,1)$ \\
\hline & Sempre & $0(0,0)$ & $0(0,0)$ & $1(2,1)$ \\
\hline \multirow[t]{3}{*}{ Dificuldade nas atividades habituais } & Nunca & $62(93,9)$ & $2(100,0)$ & $43(91,5)$ \\
\hline & Quase nunca & $3(4,5)$ & $0(0,0)$ & $2(4,3)$ \\
\hline & Algumas vezes & $1(1,5)$ & $0(0,0)$ & $2(4,3)$ \\
\hline \multirow[t]{4}{*}{ Têm avaliado a vida menos satisfatória } & Nunca & $60(90,9)$ & $2(100,0)$ & $42(89,4)$ \\
\hline & Quase nunca & $3(4,5)$ & $0(0,0)$ & $1(2,1)$ \\
\hline & Ocasionalmente & $1(1,5)$ & $0(0,0)$ & $0(0,0)$ \\
\hline & Algumas vezes & $2(3,0)$ & $0(0,0)$ & $4(8,5)$ \\
\hline
\end{tabular}


ISSN 1983-5183

\begin{tabular}{lllll}
\hline \hline Variável & & Prótese Superior & Prótese Inferior & Ambas \\
& & $\mathrm{n}(\%)$ & $\mathrm{n}(\%)$ & $\mathrm{n}(\%)$ \\
& & $\mathrm{n}=66$ & $\mathrm{n}=2$ & $\mathrm{n}=47$ \\
\hline Totalmente incapaz de realizar ativi- & Nunca & $60(90,9)$ & $2(100,0)$ & $43(91,5)$ \\
& Quase nunca & $4(6,1)$ & $0(0,0)$ & $1(2,1)$ \\
& Algumas vezes & $2(3,0)$ & $0(0,0)$ & $3(6,4)$ \\
\hline \hline
\end{tabular}

A análise do deslocamento para frente e para trás, nos diferentes tipos de próteses, revelou diferença entre o desempenho mediano para a prótese superior que foi de $99,0(95,7-100,0)$, se comparado aos pacientes que utilizam apenas a inferior, $95,0(90,0)$, e $97,0(80,0-100,0)$ para ambas as próteses, resultados considerados estatisticamente significativos $(p=0,047)$ conforme ilustra a Tabela 3.

Analisando a satisfação geral mediana dos pacientes associada à utilização das próteses superiores $99,0(96,0-100,0)$, inferiores 100,0 (100,0-100,0) e ambas 100,0 (91,0-100,0), observamos que não houve diferença estatisticamente significativa $(p=0,390)$, assim como a satisfação geral mediana associada ao gênero feminino 99,0 (96,0-100,0) e masculino $99,0(85,0-100,0)(p=0,743)$. Embora os resultados possam sugerir uma correlação negativa $\left(r_{s}=-0,82\right)$ entre a idade e 0 grau de satisfação, não houve diferença estatisticamente significativa $(p=0,388)$.

Tabela 3 - Avaliação do desempenho das próteses superior, inferior ou ambas.

\begin{tabular}{|c|c|c|c|c|}
\hline Variável* & $\begin{array}{c}\text { Prótese Superior } \\
\qquad \mathrm{n}=66\end{array}$ & $\begin{array}{l}\text { Prótese Inferior } \\
\qquad \mathrm{n}=2\end{array}$ & $\begin{array}{c}\text { Ambas } \\
\mathrm{n}=47\end{array}$ & $\mathrm{p}$ \\
\hline Desempenho & $99,0(95,7-100,0)$ & $95,0(90,0-)$ & $97,0(80,0-100,0)$ & 0,047 \\
\hline \multicolumn{5}{|c|}{ Dificuldade na mastigação } \\
\hline Pão fresco & $98,0(93,0-100,0)$ & $86,5(75,0)$ & $96,5(77,5-100,0)$ & 0,195 \\
\hline Queijo & $98,0(94,0-100,0)$ & $86,5(74,0)$ & $96,5(90,7-100,0)$ & 0,777 \\
\hline Cenoura crua & $94,5(74,0-99,0)$ & $86,5(74,0)$ & $85,0(49,0-97,0)$ & 0,193 \\
\hline Bife & $96,0(80,7-99,0)$ & $86,5(74,0)$ & $90,0(56,0-98,0)$ & 0,140 \\
\hline Maçã crua & $96,0(78,7-99,0)$ & $87,0(75,0)$ & $91,0(64,5-98,5)$ & 0,391 \\
\hline Alface & $98,0(93,5-100,0)$ & $87,5(75,0)$ & $97,0(91,0-99,2)$ & 0,831 \\
\hline
\end{tabular}

*Mediana (Intervalo interquartil)

A análise entre a escolaridade dos participantes e a renda mensal mostrou que $62,0 \%$ ( $n=49)$ que possuíam ensino fundamental incompleto tinham renda de até um salário mínimo mensal, enquanto que $27,8 \%(\mathrm{n}=22)$ não possuíam renda.

Em relação à análise da escolaridade com a satisfação geral mediana, o estudo salientou, para aqueles com ensino fundamental incompleto, que foi 99,0 (96,0-100,0), ilustrado na Tabela 4. 
ISSN 1983-5183

Tabela 4 - Associação da escolaridade com renda mensal e satisfação geral.

\begin{tabular}{|c|c|c|c|c|c|c|}
\hline Variável* & $\begin{array}{c}\text { Nunca } \\
\text { estudou } \\
\text { n(\%) } \\
n=9\end{array}$ & $\begin{array}{c}\text { Ensino fund. } \\
\text { incompleto } \\
\begin{array}{c}n(\%) \\
n=79\end{array}\end{array}$ & $\begin{array}{c}\text { Ensino fund. } \\
\text { completo } \\
\begin{array}{c}n(\%) \\
n=12\end{array}\end{array}$ & $\begin{array}{c}\text { Ensino médio } \\
\text { incompleto } \\
\begin{array}{c}n(\%) \\
n=8\end{array}\end{array}$ & $\begin{array}{l}\text { Ensino médio } \\
\text { completo } \\
\begin{array}{c}\mathrm{n}(\%) \\
\mathrm{n}=7\end{array}\end{array}$ & $p$ \\
\hline Renda mensal & & & & & & \\
\hline Não possui renda & $2(22,2)$ & $22(27,8)$ & $3(25,0)$ & $3(37,5)$ & $0(0,0)$ & \\
\hline Até um salário mínimo & & & & & & \\
\hline De 1 a 2 salários & $\begin{array}{l}7(77,8) \\
0(0,0)\end{array}$ & $49(62,0)$ & $4(33,3)$ & $3(37,5)$ & $\begin{array}{l}6(85,7) \\
1(14,3)\end{array}$ & 0,070 \\
\hline $\begin{array}{l}\text { Satisfação geral median } \\
\text { (Intervalo Interquartil) }\end{array}$ & $\begin{array}{l}100,0 \\
(95,0- \\
100,0)\end{array}$ & $\begin{array}{c}99,0(96,0- \\
100,0)\end{array}$ & $\begin{array}{c}100,0(91,0- \\
100,0)\end{array}$ & $\begin{array}{c}99,0(84,7- \\
100,0)\end{array}$ & $\begin{array}{c}100,0(85,0- \\
100,0)\end{array}$ & 0,986 \\
\hline
\end{tabular}

Na análise das principais questões do questionário EVA, a habilidade com a mastigação associada às mulheres $97,0(86,0-99,0)$ e homens $93,5(67,0-98,0)$, não foi considerada estatisticamente significativa $(p=0,125)$. Quanto à satisfação com suas condições bucais, o gênero feminino chegou a 99,0 $(86,0-100,0)$ e masculino a 89,0 (70,7-100,0); embora os resultados possam sugerir diferença entre essas medianas, a significância foi considerada estatisticamente limítrofe $(p=0,066)$, conforme ilustra a Tabela 5.

Tabela 5 - Principais questões sociais, biológicas e psicológicas do EVA, associadas ao gênero.

\begin{tabular}{|c|c|c|c|}
\hline Variável* & $\begin{array}{c}\text { Gênero } \\
\text { Feminino } \mathrm{n}=79\end{array}$ & $\begin{array}{c}\text { Gênero } \\
\text { Masculino } n=36\end{array}$ & $\mathrm{p}$ \\
\hline Facilidade com limpeza & $100,0(97,0-100,0)$ & $100,0(98,25-100,0)$ & 0,407 \\
\hline Satisfação geral & $99,0(96,0-100,0)$ & $99,0(85,0-100,0)$ & 0,743 \\
\hline Facilidade para falar & $97,0(88,0-100,0)$ & $95,0(86,5-100,0)$ & 0,689 \\
\hline Conforto & $98,0(88,0-100,0)$ & $97,0(84,2-99,7)$ & 0,443 \\
\hline Estética & $98,0(94,0-100,0)$ & $98,5(94,0-100,0)$ & 0,834 \\
\hline Satisfação com a retenção & $97,5(84,7-100,0)$ & $90,5(79,2-99,0)$ & 0,162 \\
\hline Habilidade com mastigação & $97,0(86,0-99,0)$ & $93,5(67,0-98,0)$ & 0,125 \\
\hline
\end{tabular}


Cristiano DP, Collodel A, Ceretta LB, Simões PW, Ceretta RA, Sonego FGF. Avaliação do desempenho e satisfação dos usuários de próteses totais mucossuportadas fornecidas pelo Sistema Único de Saúde em município catarinense. Rev. Odontol. Univ. Cid. São Paulo 2018 abr/jun 30(2) 116-31

ISSN 1983-5183

\begin{tabular}{|c|c|c|c|}
\hline \multirow[t]{2}{*}{ Variável* } & Gênero & Gênero & $\mathrm{p}$ \\
\hline & Feminino $n=79$ & Masculino $\mathrm{n}=36$ & \\
\hline Comida bem mastigada antes de engolir & $98,0(85,0-99,0)$ & $95,0(71,5-99,0)$ & 0,207 \\
\hline $\begin{array}{l}\text { Satisfação com suas condições de saúde } \\
\text { bucal }\end{array}$ & $99,0(86,0-100,0)$ & $89,0(70,7-100,0)$ & 0,066 \\
\hline
\end{tabular}

*Mediana (intervalo interquartil)

O objetivo principal do nosso estudo foi analisar a satisfação e o desempenho dos usuários de próteses totais confeccionadas pelo SUS. Foram avaliados 115 pacientes, sendo 57,4\% com próteses totais superiores, 1,7\% com inferiores e 40,9\% com ambas. Quando comparados os resultados obtidos com dados descritos na literatura, esses achados são semelhantes aos encontrados em um estudo transversal, com 120 pacientes, divididos em 59,18\% com próteses superiores e 40,82\% com inferiores e analisou-se a satisfação sobre as próteses fornecidas pelo SUS de um município catarinense ${ }^{12}$. Em outro estudo realizado em Santa Maria/RS, com amostra de 50 usuários de próteses totais, sendo distribuídas $46 \%$ superiores, apenas $10 \%$ inferiores e 44\% ambas as próteses, verificaram-se a satisfação e o impacto desse tipo de reabilitação na vida dos pacientes ${ }^{13}$. No nosso estudo, $68,7 \%$ dos participantes eram do gênero feminino e apenas $31,3 \%$ do masculino, prevalência feminina semelhante observada em outros estudos ${ }^{13,14,15}$.

O perfil socioeconômico foi verificado e $60,0 \%$ possuem renda mensal de até um salário mínimo e $26,1 \%$ não possuem nenhum tipo de renda. Ao analisar o nível de escolaridade, a maioria dos contemplados com as próteses do nosso estudo (68,7\%) tem o ensino fundamental incompleto e 10,4\% ensino fundamental completo. Esses dados foram caracterizados como um perfil amostral relativamente carente e idoso; apesar da idade mediana ser de 59,0 anos, pode-se associar esses achados com as características literárias do edentulismo. Um estudo com 50 usuários do SUS, em fase de tratamento reabilitador com prótese total e idade mediana de 48,0 anos, apontou a perda dentária como consequência da falta de informação sobre a saúde bucal e a dificuldade de acesso ao serviço público. Verificou-se, também, que mesmo os que têm acesso aos serviços de saúde bucal aderiram a essa condição por ser única alternativa de custo, tendo a condição econômica como papel fundamental para o edentulismo, geralmente associado a fatores psicossociais e culturais. Afirmou-se, também, que aqueles que apresentaram menor nível de escolaridade e moradores de zonas rurais têm uma probabilidade maior de tornarem-se edêntulos ${ }^{16}$. Observou-se predomínio da utilização de próteses dentárias em idosos, com ensino fundamental incompleto ou analfabeto e renda mensal de um salário mínimo ${ }^{15}$. No entanto, em outro estudo, pacientes com ensino superior apresentaram efeito positivo sobre a qualidade de vida relacionada à saúde bucal, pelo fato de compreenderem melhor suas condições bucais e possuírem maior conhecimento sobre os cuidados com a saúde ${ }^{17}$.

Impactos negativos referentes à dor, desconforto e inabilidade psicológica são observados em pacientes com perda dental, na utilização de próteses totais inadequadas ${ }^{2}$, que, também, podem influenciar na autoestima desses indivíduos ${ }^{13}$. No entanto, a reabilitação deve proporcionar a integração desses pacientes ao convívio social e não apenas devolver as funções perdidas como consequência do edentulismo ${ }^{14}$.

Os resultados para a limitação funcional, que avaliou a dificuldade na pronúncia de palavras, foram positivos, sendo que, entre os participantes que utilizavam próteses superiores, $75,8 \%$ nunca encontraram 
Cristiano DP, Collodel A, Ceretta LB, Simões PW, Ceretta RA, Sonego FGF. Avaliação do desempenho e satisfação dos usuários de próteses totais mucossuportadas fornecidas pelo Sistema Único de Saúde em município catarinense. Rev. Odontol. Univ. Cid. São Paulo 2018 abr/jun 30(2) 116-31

ISSN 1983-5183

problema, assim como 100,0\% dos que utilizavam somente a inferior e 70,2\% para ambas as próteses. Nesse contexto, resultados semelhantes são encontrados, onde pacientes que utilizaram próteses superiores e inferiores, respectivamente, $87,8 \%$ e $83 \%$ não apresentaram dificuldades na pronúncia de palavras ${ }^{12}$. Entretanto, observou-se que $12 \%$ dos usuários de próteses totais sempre apresentaram dificuldade em pronunciar as palavras ${ }^{13}$, assim como nos achados deste outro estudo ${ }^{10}$, onde 49 pacientes foram reabilitados com próteses totais confeccionadas pelos métodos convencionais ou duplicados e aplicado o questionário semelhante ao utilizado no nosso estudo; porém, um antes e outro após um mês do tratamento reabilitador mostraram que o grupo convencional apontou dificuldades na capacidade de falar, podendo ser explicado pelo fato da fala ser uma função complexa que exija um tempo maior de adaptação das novas próteses ${ }^{10}$. Esses resultados apontados são superiores aos encontrados na nossa pesquisa, onde apenas 3,0\% relataram tal dificuldade na pronúncia com utilização de prótese superior e 2,1\% para superior e inferior. Pode-se averiguar que, no nosso estudo, a maioria dos pacientes entrevistados, está satisfeita com suas próteses quanto à pronúncia de palavras, como descrito inicialmente.

A maioria dos participantes não encontrou dificuldade em sentir o gosto dos alimentos, mesmo para próteses superiores $83,3 \%$ não relataram problema, seguidos de $100 \%$ dos que utilizam inferiores e $70,2 \%$ para ambas. Assim como, em outros estudos analisados, 91,8\% dos que utilizaram prótese superior e $88,9 \%$ as inferiores não notaram alteração em seu paladar ${ }^{12}$, e $62 \%$ nunca observaram determinada piora em relação ao gosto dos alimentos com a utilização de próteses totais ${ }^{13}$, resultados semelhantes aos encontrados em nosso estudo.

Os resultados obtidos segundo o questionamento sobre dor na boca devido à utilização das próteses mostraram que $42,4 \%$ quase nunca sentiam dor quando utilizavam próteses superiores e, para os que utilizam ambas as próteses, $46,8 \%$ nunca sentiram dor. No entanto, 3,0\% daqueles que utilizam próteses superiores relataram sempre sentir dor, seguidos de $6,4 \%$ que utilizam ambas as próteses e $50 \%$ dos usuários apenas de prótese total inferior também sempre sentiam dor. No estudo com 153 pacientes que procuraram reabilitação protética no período entre 2009 e 2011, observou-se, por meio das respostas obtidas, após um mês de utilização, que a reabilitação com prótese total apresentou impacto negativo no quesito dor e desconforto, assim como perante a utilização de outras próteses removíveis ${ }^{18}$. Outro estudo mostrou que $8 \%$ sempre apresentaram dor, e $24 \%$ deles consideraram a utilização das próteses totais desconfortáveis ${ }^{13}$. No nosso estudo $50 \%$ dos participantes que utilizam próteses inferiores consideraram sempre desconfortáveis, seguidos de 8,5\% com ambas as próteses e apenas 3\% daqueles com próteses superiores relataram tal desconforto.

Quando questionados sobre a alimentação ser insatisfatória, 68,2\% dos usuários de próteses superiores nunca tiveram essa insatisfação, assim como aqueles que utilizam ambas as próteses (48,9\%). Em um estudo transversal com 150 pacientes idosos, usuários de próteses totais há 5 anos, na comparação entre os grupos, referente à dieta ser insatisfatória, os reabilitados com prótese total mostraram-se insatisfeitos quando comparados a outros tipos de prótese em que foram reabilitados ${ }^{19}$. Quando questionados sobre o fato de terem que interromper alguma refeição por causa da utilização de próteses, 72,7\%, que utilizam apenas a superior, afirmaram nunca terem interrompido, seguidos de $53,2 \%$ dos que usam ambas as próteses, que também nunca precisaram interromper as refeições. Corroborando o nosso estudo, pôde-se observar que $66 \%$ dos participantes também nunca necessitaram interromper as refeições por causa das próteses totais ${ }^{13}$.

Sobre o desempenho das próteses superiores, inferiores e ambas, no questionário EVA, observou-se significância estatística $(p=0,047)$ entre esses diferentes grupos, com relação à satisfação dos pacientes, 
ISSN 1983-5183

no deslocamento para frente e para trás, na utilização das próteses. Porém a satisfação na mastigação dos alimentos com diferentes consistências não mostrou diferença estatística, mesmo estando interligada ao desempenho das próteses. Sendo assim, essa diferença pode ser entendida pela dificuldade de adaptação às novas próteses e também por ser considerada uma amostra pequena; outros estudos devem ser realizados para confirmarem esse achado ${ }^{18}$. No entanto, um estudo com 32 pacientes reabilitados com prótese total e prótese parcial removível, apresentando metodologia semelhante ao nosso estudo, analisou o grau de satisfação dos pacientes reabilitados e foi possível observar que os reabilitados com próteses totais apresentaram maior satisfação referente à mastigação quando comparados ao outro grupo $(p=0,01)^{20}$.

Um estudo com uma amostra de 99 participantes, que analisou a expectativa e satisfação dos pacientes reabilitados com próteses totais, concluiu que a satisfação referente à mastigação está relacionada ao número de ajustes realizados posteriores à entrega das próteses ${ }^{21}$. Essa diferença no desempenho dos diferentes tipos de próteses pode estar relacionada ao fato de a utilização da prótese total superior apresentar uma superfície de área basal maior, associada a uma boa estrutura anatômica facilitando a reabilitação e adaptação, diferente das inferiores que têm uma área basal menor e apresenta algumas estruturas que dificultam a sua estabilidade, como a língua, por exemplo, facilitando o seu deslocamento ${ }^{20,22}$. Pode-se identificar esse problema quando analisados os achados do nosso estudo, sendo que $9,6 \%$ relataram que a prótese inferior não deu certo e não foi possível sua utilização, assim como não procuraram o atendimento novamente para realizar os devidos ajustes.

Analisando-se a satisfação geral, esta apresentou bons resultados, relacionados ao gênero e não apresentou diferença estatisticamente significativa $(p=0,743)$ e, apesar dos resultados sugerirem uma correlação negativa com a idade e o grau de satisfação, também não houve diferença estatística $(p=0,388)$, mas observou-se que a satisfação foi diretamente proporcional ao aumento da idade, ou seja, quanto maior a idade, maior o grau de satisfação. Este estudo apontou as mulheres com uma satisfação elevada após a conclusão do tratamento, principalmente no quesito estético, em comparação aos homens ${ }^{21}$, resultado divergente do nosso estudo que não apresentou diferença estatisticamente significativa $(p=0,834)$ para os gêneros, quando analisada a satisfação referente à estética. A satisfação dos pacientes reabilitados com próteses totais pode estar relacionada com a capacidade de aceitação da sua condição bucal, do tratamento proposto e das suas limitações perante o tratamento, mais do que em relação à técnica utilizada ${ }^{10}$. Outro estudo aponta o nível de satisfação como multifatorial, envolvendo vários critérios como adaptação pelo paciente e a técnica ${ }^{21}$.

A avaliação da experiência, a expectativa e a satisfação dos pacientes foram o objetivo do estudo, com 100 participantes, para reabilitação com prótese total, e se observou que o nível educacional pode influenciar na expectativa dos pacientes referente ao conforto, sendo que avaliaram com maiores índices quando comparados àqueles com escolaridade maior, onde a expectativa pela reabilitação está totalmente interligada com a satisfação após a finalização do tratamento ${ }^{23}$. No nosso estudo, pôde-se destacar que a escolaridade não afetou a satisfação geral dos participantes e mesmo com a diferença entre os grupos, não apresentou significância estatística $(p=0,986)$.

Por meio deste estudo pode-se verificar que, apesar das limitações encontradas por meio da reabilitação com próteses totais, a maioria dos pacientes encontra-se satisfeitos em todos os quesitos abordados pelos dois questionários. As condições das próteses não foram relevantes para afetar o grau de satisfação. Mesmo com algumas limitações, a satisfação em receber as próteses totais mucossuportadas pelo SUS foi observada e ficou caracterizada como primordial em função de estarem totalmente edêntulos 
ISSN 1983-5183

e sem recursos financeiros para o tratamento adequado.

Sendo assim, mais estudos, envolvendo essa metodologia, devem ser realizados para analisar a satisfação e desempenho dos usuários reabilitados com as próteses totais, confeccionadas pelo LRPD do SUS. Outros tipos de próteses, também fornecidas pelo sistema, necessitam de avaliações para que seja fortalecida essa ação, possibilitando a reabilitação para todos os necessitados com qualidade.

\section{CONCLUSÃO}

O presente estudo revelou aceitação quanto ao desempenho das próteses avaliadas. Na análise do deslocamento para frente e para trás, constatou-se diferença estatisticamente significativa para reabilitação com prótese superior, inferior ou ambas.

Verificou-se que os usuários do SUS que receberam próteses totais mucossuportadas, no município sul catarinense, em estudo, estão satisfeitos com a reabilitação.

\section{REFERÊNCIAS}

1. REISSMANN DR, John MT, Schierz O, Kriston L, Hinz A. Association between perceived oral and general health. Journal of dentistry 2013 Jul;41(7):581-9.

2. SILVA MES, Villaça ÊL, Magalhães CS, Ferreira EF. Impacto da perda dentária na qualidade de vida. Ciênc saúde coletiva 2010 maio;15(3):841-50.

3. PIUVEZAM G, Ferreira AAA, Alves MSCF. Enfrentando as perdas dentárias na terceira idade: um estudo de representações sociais. Cad Saúde Colet 2006 14(4):597-614.

4. BRASIL. Ministério da Saúde. SB Brasil 2010: pesquisa nacional de saúde bucal: resultados principais. Brasília: Ministério da Saúde; 2012 [Acesso em: 25 jun. 2018]; Disponível em: http://bvsms.saude.gov.br/bvs/publicacoes/pesquisa nacional saude bucal.pdf.

5. BRASIL. Saúde bucal terá R\$36,2 mi para investimento em laboratórios de próteses dentárias. 2013 [Acesso em: 25 jun. 2018]; Disponível em: http://www.brasil.gov.br/editoria/saude/2013/09/saude-bucal-tera-r-36-2-mi-para-investimento-em-laboratorios-de-proteses-dentarias.

6. COLUSSI CF, Nicke IDA, Calvo MCM, Caetano JC, Silva ACB. Análise da evolução da produção de procedimentos odontológicos de média e alta complexidade na rede de serviços públicos em Santa Catarina. Cad Saúde Colet 2009 out.;17(4):939-50.

7. INSTITUTO Brasileiro de Geografia e Estatística. Análise da população estimada no município de Içara-Santa Catarina. Santa Catarina: IBGE; 2014 [Acesso em: 26 jun. 2018]; Disponível em: https://cidades.ibge.gov.br/brasil/sc/icara/panorama.

8. GABARDO MCL, Moysés ST, Moysés SJ. Autopercepção de saúde bucal conforme o Perfil de Impacto da Saúde Bucal (OHIP) e fatores associados: revisão sistemática. Rev panam salud pública $=$ Pan am j public health 2013 jun.;33(6):439-45.

9. AWAD MA, Feine JS. Measuring patient satisfaction with mandibular prostheses. Community dentistry and oral epidemiology 1998 Dec;26(6):400-5. 
ISSN 1983-5183

10. ELLIS JS, Pelekis ND, Thomason JM. Conventional rehabilitation of edentulous patients: the impact on oral health-related quality of life and patient satisfaction. Journal of prosthodontics : official journal of the American College of Prosthodontists 2007 Jan-Feb;16(1):37-42.

11. REGIS RR. Método simplificado versus convencional de confecção de próteses totais para aplicação na saúde pública. Parte II: qualidade de vida associada à saúde bucal, satisfação do paciente, qualidade das próteses [Tese]. Ribeirão Preto: Faculdade de Odontologia de Ribeirão Preto; 2012.

12. VERONEZ FC, Sônego FGF, Ceretta RA, Zaccaron S, Ceretta LB. User satisfaction with complete dentures made by the public network of a city in southern Santa Catarina. RGO, Rev Gaúch Odontol 2014 jun.;62(2):123-7.

13. ROCHA FR, Perlin J, Guerino P, Guimarães MB. Impacto do uso de próteses totais na qualidade de vida de idosos. Rev Contexto Saúde, ljuí 2011 Jan.-Jun. ;10(20):

14. COSTA APS, Machado FCA, Pereira ALBP, Carreiro AFP, Ferreira MÂF. Qualidade técnica e satisfação relacionadas às próteses totais. Ciênc saúde coletiva 2013 fev.;18(2):45360 .

15. MIRANZI MAS, Amuí MM, Iwamoto HH, Tavares DMS, Pinheiro SA, Coimbra MAR. Uso da prótese dentária entre idosos: um problema social. REFACS 2015 3(1):4-11.

16. SILVA MES, Magalhães CS, Ferreira EF. Perda dentária e expectativa da reposição protética: estudo qualitativo. Ciênc saúde coletiva 2010 maio;15(3):813-20.

17. ZINI A, Sgan-Cohen HD. The effect of oral health on quality of life in an underprivileged homebound and non-homebound elderly population in Jerusalem. Journal of the American Geriatrics Society 2008 Jan;56(1):99-104.

18. MONTERO J, Castillo-Oyague R, Lynch CD, Albaladejo A, Castano A. Self-perceived changes in oral health-related quality of life after receiving different types of conventional prosthetic treatments: a cohort follow-up study. Journal of dentistry 2013 Jun;41(6):493-503.

19. MARTIN-ARES M, Barona-Dorado C, Guisado-Moya B, Martinez-Rodriguez N, Cortes-Breton-Brinkmann J, Martinez-Gonzalez JM. Prosthetic hygiene and functional efficacy in completely edentulous patients: satisfaction and quality of life during a 5-year follow-up. Clinical oral implants research 2016 Dec;27(12):1500-5.

20. BELONI WB, Vale HF, Takahashi JMFK. Avaliação do grau de satisfação e qualidade de vida dos portadores de prótese dental RFO UPF 2013 mai.-ago.;18(2):160-4.

21. SANTOS BF, Santos MB, Santos JF, Marchini L. Patients' evaluations of complete denture therapy and their association with related variables: a pilot study. Journal of prosthodontics : official journal of the American College of Prosthodontists 2015 Jul;24(5):3517.

22. CHEN Y-F, Yang Y-H, Chen J-H, Lee H-E, Lin Y-C, Ebinger J, et al. The impact of complete dentures on the oral health-related quality of life among the elderly. J Dent Sci 2012 
ISSN 1983-5183

7(3):289-95.

23. GASPAR MG, Santos MB, Santos JF, Marchini L. Correlation of previous experience, patient expectation and the number of post-delivery adjustments of complete dentures with patient satisfaction in a Brazilian population. Journal of oral rehabilitation 2013 Aug;40(8):590-4.

RECEBIDO EM 22/08/2017

ACEITO EM 04/04/2018 